\title{
Food science research contribution to the prevention of SARS CoV-2 infection and COVID-19 pandemic treatment
}

\author{
Jack N. Losso
}

School of Nutrition and Food Sciences, Louisiana State University, Baton Rouge, LA 70803, USA. Tel: 225-578-3883; E-mail: jlosso@1su.edu

DOI: $10.31665 /$ JFB.2020.11234

Received: September 26, 2020; Revised received \& accepted: September 30, 2020

Citation: Losso, J.N. (2020). Food science research contribution to the prevention of SARS CoV-2 infection and COVID-19 pandemic treatment. J. Food Bioact. 11: 11-12.

\begin{abstract}
SARS CoV-2 virus is primed by proteases before it attaches to host cells and causes the Coronavirus Diseases-2019 (COVID-19). The proteases that activate SARS-CoV-2 virus can be inhibited by proteins naturally occurring in human plant foods such as legumes. Food science contribution to the fight against SARS-CoV-2 infection and COVID-19 needs to extend beyond the traditional involvement in developing and securing high quality foods to include investigating foods rich in plant protease inhibitors as novel foods for SARS-CoV-2 and COVID-19 prevention.
\end{abstract}

Keywords: SARS-CoV-2; COVID-19; Prevention; Plant protease inhibitors.

Food Science contribution to the SARS-CoV-2 infection and COVID-19 pandemic is not limited to rinsing food and cleaning food processing facilities surfaces, the global food chain supply, consumer behavior, and food security. Food scientists do much more to help fight SARS-CoV-2 infectivity and COVID-19 with the quality of foods being developed, cooked, and putting on the table. The time for food scientists and food research sponsors to contribute further to the fight against SARS-CoV-2 is now and cannot wait and here is why.

Social distancing, confinement, mask wearing, and regular hand washings are among the best practices to prevent SARS$\mathrm{CoV}-2$ infection. When these protective measures fail, SARS$\mathrm{CoV}-2$ is transmitted from an infected individual to another. Here is what we know about the virus. SARS-CoV-2 uses proteases to prime the virus and allow it to attach to the receptor angiotensin converting enzyme 2 (ACE2) that is located on nasopharyngeal, lung, heart, and intestine cell surfaces. If inhibited to bind to the cell receptor, the virus vanishes. If successful to attach after being primed/activated or after endocytosis the virus penetrates the cell, replicates, and causes COVID-19. The severity of COVID-19 can be amplified by obesity, diabetes, hypertension, cardiovascular disease, kidney disease, asthma, or allergies because individuals with these conditions have impaired endothelial cells and elevated levels of serum proteases that can catalyze SARS-CoV-2 activation. Obesity affects about $30 \%$ of the US population (about 110 million) while diabetes affects 34.2 million Americans. In 2017, nearly half of adults in the US (about 108 million) had hypertension and only about $24 \%$ of these individuals had their conditions under control. Obesity affects $61 \%$ of Canadians, $5-6 \%$ of Chinese. Cardiovascular disease is the number one killer in the world (www.cdc.gov).

At current level of understanding, proteases that prime the SARS-CoV-2 virus for entry into the host and the coagulopathy that COVID-19 is causing in severely infected individuals are two targets that those involved in finding a non-vaccine or antiviral cure for COVID-19 are focusing on (Drak Alsibai, 2020; Ji et al., 2020; Mucha et al., 2020). These two targets can well be addressed by the food that we consume. Plant foods including legumes (soybeans, lima beans, dry beans, chickpeas, adzuki beans, mung beans), potato, sweet potato, yams, summer and winter squash, banana, sunflower seeds, pumpkin seeds, moringa leaves, and bitter gourd seeds are excellent sources of protease inhibitors that are bioavailable and can prevent SARS-CoV-2 to attach to the host cells (Srikanth and Chen, 2016). Some of these plant foods such as winter squash are also excellent sources of protease inhibitors that are bioavailable and can help prevent blood clotting, a death causing factor in COVID-19 (Otlewski and Krowarsch, 1996). When minimally processed, these foods can serve as good sources of protease inhibitors that can deliver these bioactives daily and may help individuals fight SARS-CoV-2 in- 
fection (Otlewski and Krowarsch, 1996; Srikanth and Chen, 2016; Theoharides, 2020).

The rate of SARS-CoV-2 infectivity and COVID-19-induced mortality appears to be low in countries where minimally processed foods rich in protease inhibitors are consumed regularly and in sizable quantities. Populations in countries that consume high volumes of soymilk and soy products rich in protease including Hong Kong, Singapore, Vietnam, Laos, Cambodia, and Macau have a low rate of SARS-CoV-2 infectivity and COVID-19 related deaths https://coronavirus.jhu.edu/map.html. Countries that consume high volumes of plant foods rich in protease inhibitors such as dry beans and cook the beans by boiling at temperature below $100{ }^{\circ} \mathrm{C}$ have a low rate of SARS-CoV-2 infectivity and COVID-19 related death. Rwanda, Burundi, and the Nicoya Region of Costa Rica top the list https://coronavirus.jhu.edu/ map.html. Obesity is one of the premier risk factors for severe COVID-19 outcomes. Plant protease inhibitors may help lose weight and can also inhibit mast cell overactivation (Hill et al., 1990). Adopting plant-based diet and cuisine that use minimally cooked foods may add additional barriers against the severity of COVID-19.

The time is ripe for food scientists to focus on foods rich in protease inhibitors and other bioactive compounds that can significantly affect the infectivity of SARS-CoV-2. The focus can be on basic concept such as minimally processed foods, boiled foods, and safe and optimal delivery of protease inhibitors, anti-angiogenic foods, or other health enhancing dietary bioactives to the circulation well before SARS-CoV-2 infection and help individuals, especially those with risk factors such obesity and diabetes, to fight SARS-CoV-2 attachment to the host or COVID-19.

Performing research on antiviral foods and working along with virologists is the best contribution food scientists can make to help combat SARS-CoV-2 infection and COVID-19 pandemic.

\section{References}

Drak Alsibai, K. (2020). Expression of angiotensin-converting enzyme 2 and proteases in COVID-19 patients: A potential role of cellular FURIN in the pathogenesis of SARS-CoV-2. Med. Hypotheses 143: 109893. doi:10.1016/j.mehy.2020.109893.

Hill, A.J., Peikin, S.R., Ryan, C.A., and Blundell, J.E. (1990). Oral administration of proteinase inhibitor II from potatoes reduces energy intake in man. Physiol. Behav. 48(2): 241-246. doi:10.1016/00319384(90)90307-p.

Ji, H.L., Zhao, R., Matalon, S., and Matthay, M.A. (2020). Elevated Plasmin(ogen) as a Common Risk Factor for COVID-19 Susceptibility. Physiol. Rev. 100(3): 1065-1075. doi:10.1152/physrev.00013.2020.

Mucha, S.R., Dugar, S., McCrae, K., Joseph, D., Bartholomew, J., Sacha, G.L., and Militello, M. (2020). Coagulopathy in COVID-19: Manifestations and management. Cleve. Clin. J. Med. 87(8): 461-468. doi:10.3949/ ccjm.87a.ccc024.

Otlewski, J., and Krowarsch, D. (1996). Squash inhibitor family of serine proteinases. Acta Biochim. Pol. 43(3): 431-444.

Srikanth, S., and Chen, Z. (2016). Plant Protease Inhibitors in Therapeutics-Focus on Cancer Therapy. Front Pharmacol. 7: 470. doi:10.3389/ fphar.2016.00470.

Theoharides, T.C. (2020). COVID-19, pulmonary mast cells, cytokine storms, and beneficial actions of luteolin. Biofactors 46(3): 306-308. doi:10.1002/biof.1633. 